\title{
Phenacetin Crystallization: Cooling Regimes and Crystal Morphology
}

\author{
Humphrey A. Moynihan and Dawn M. Kelly \\ Dept. of Chemistry / Analytical and Biological Chemistry Research Facility, \\ University College Cork, Cork \\ Ireland
}

\section{Introduction}

Phenacetin crystallizations were undertaken to gain an insight into how to better control the various parameters of a crystallization process. Good control of the crystallization process allows the design of experiments to control certain characteristics of the final crystal product. These include crystal morphology, phase, particle size and crystal size distribution (CSD). These physical attributes of a crystal population are thought to be achieved through the control of the nucleation and growth of crystals during the crystallization process. One of the first attempts to control crystallization processes was proposed by Griffiths (Griffiths, 1925), who suggested the idea of "controlled cooling" in batch crystallizations by maintaining the supersaturation in the metastable region in order to improve the product CSD. Garside et al. performed experimental studies employing this concept of controlled cooling in crystallization processes, which included simple strategies such as isothermal operation and linear cooling (Garside et al., 1972). Since these early crystallization control approaches, there have been many strategies that indirectly affect the CSD of a crystallization process, often involving a change in temperature or the addition of an antisolvent during a pre-defined timescale so as to follow a given supersaturation profile in the phase diagram. These profiles are obtained using simple trial-and-error experimentation or more complex model-based or direct-design approaches (Abu Bakar et al., 2009a). Woo et al. proposed an adaptive concentration control strategy that employs the measurement of the number of particle counts per unit time provided by in situ laser backscattering in order to detect the onset of nucleation and adapt the operating curve accordingly (Woo et al., 2009). In a move towards new strategies for directly affecting the CSD of a crystallisation process, Abu Baker et al. (Abu Bakar et al., 2009a) used a model-free approach to crystallization control known as 'direct nucleation control' (DNC), in which the number of counts measured by an in situ laser probe is directly controlled using a feedback control strategy. An alternative strategy is to chart an oscillating cooling regime within the metastable zone of the crystallization, in which the overall cooling trend drives crystal growth while the temperature fluctuations provided by the oscillating cool allows partial dissolution (Abu Bakar et al., 2009b). The result should be an equalizing of crystal size and shape. Both of these approaches were utilized in the present study. Phenacetin was selected as the subject for this study as it is an Active Pharmaceutical Ingredient - now withdrawn (Prescott, 1980) - and is not known to be crystal polymorphic (Hansen, 2006). 
This chapter will present work on phenacetin crystallization using two forms of modified cooling regimes in an attempt to directly affect the product CSD of the crystallization process. We begin with a cooling regime using turbidity counts as a termination clause for each cooling and heating step. We then move to a more in-depth study in Section 3.2, where the cyclic cooling paths that had predefined temperature parameters were used, ensuring that a cyclic temperature profile was maintained within the limits of the metastable zone width (MSZW). This temperature profile was coupled with a seeding regime where each experiment was seeded with crystals that were sieved to give specific size ranges of less than $150 \mu \mathrm{m}$ (fine particles), between 150-300 $\mu \mathrm{m}$ (medium particles) and over $500 \mu \mathrm{m}$ (coarse particles). The objective of both of these types of modified cooling regimes is to provide a narrower range of crystal sizes and shapes than would otherwise be obtained by a simple linear cooling regime.

\section{Experimental methods}

Phenacetin was purchased from Sigma-Aldrich. GPR grade absolute ethanol was purchased from Carbon Group Chemical Ltd. (Cork, Ireland) and was used in all of the experiments. Ethanol was chosen as a solvent for these experiments because the solubility of phenacetin in ethanol varies significantly over the temperature range $10^{\circ} \mathrm{C}$ to $30^{\circ} \mathrm{C}$, allowing for the development of a practical crystallization method. The determination of phenacetin solubility and metastable zone width is described below. Phenacetin crystallizes cleanly from ethanol to give well-formed needles which are largely free from the inclusions of other defects.

A HEL Autolab jacketed reactor vessel equipped with a HEL Lasertrack in situ particle sizing laser probe was used to monitor the presence and size of phenacetin crystals on a $1 \mathrm{~L}$ scale. The laser wavelength was $795 \mathrm{~nm}$. It is possible to get readings of particles as small as $0.5 \mu \mathrm{m}$. A PTFE PT100 thermocouple gave in situ temperature measurements of the crystallization medium. The temperature of the jacket fluid (Huber DW-Therm thermal fluid, operating range $-90^{\circ} \mathrm{C}$ to $200{ }^{\circ} \mathrm{C}$ ) was controlled by a Huber Unistat 815 circulation thermostat. The system was entirely controlled from one PC using HEL WinISO software, allowing the control of stirring rates, the addition of material (the pumping rate and mass pumped), and the heating parameters (heating or cooling rates, reactor vessel temperature set point, jacket fluid temperature set point). The software allowed the pre-programming of crystallization regimes containing as many separate steps as required, e.g., multiple heat/cool steps, solvent addition steps, etc. The steps could be programmed to terminate and move to a next step upon reaching a specified set point, such as the reactor temperature or the number of particles observed.

Crystal habits were observed using a Nikon Polarizing Microscope Eclipse 50i POL and photomicrographs were taken on a Nikon Digital Sight DS-Fi1 digital camera. Figure 1 shows a schematic representation of the experimental setup for phenacetin crystallization experiments.

In the experiments described below, in which seeding was used, crystals obtained from water were used as seed crystals because they typically have a plate-like habit. The platelike habit provides a greater surface area for growth and so is more suitable for seeds than the needle-like habit obtained from ethanol. The crystals formed from water also have 
rougher surfaces than the ethanol-derived needles, again assisting crystal growth. In addition, any differences in habit after the crystallization of phenacetin from ethanol using these seed crystals would assist in the determination of whether growth or nucleation dominated these processes. The seed crystals were sieved to give the size ranges described below using a set of U.S.A. standard testing sieves (Avantech Manufacturing) in mesh sizes of $150 \mu \mathrm{m}, 300 \mu \mathrm{m}$ and $500 \mu \mathrm{m}$. The seeds were added to crystallisations in an ethanol slurry using seed crystals with a weight in grams equal to $20 \%$ of the total batch concentration.

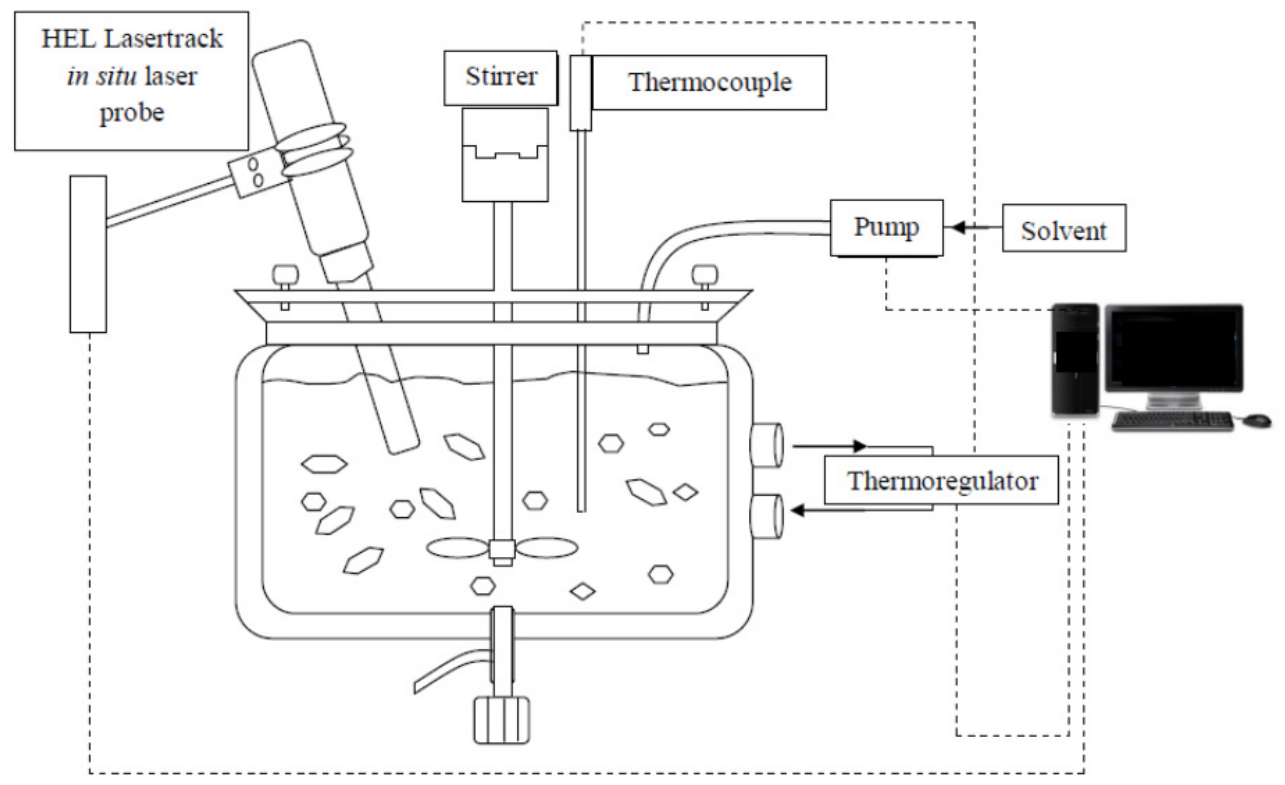

Fig. 1. A schematic representation of the experimental setup for phenacetin crystallization experiments.

Only one crystalline form of phenacetin has been reported to date (Hansen, 2006). To check that no new polymorphs or solvates of phenacetin were obtained during these experiments, powder X-ray diffraction (PXRD) patterns were recorded for all of the samples and compared with the theoretical pattern generated from the structure reported by Hansen et al. (Hansen, 2006). PXRD was performed at an ambient temperature using a Stoe Stadi MP diffractometer operating in transmission mode with a linear PSD detector, with an anode current of $40 \mathrm{~mA}$, an accelerating voltage of $40 \mathrm{kV}$ and $\mathrm{Cu} \mathrm{Ka}_{1} \mathrm{X}$-radiation $(\lambda=1.5406 \AA)$, typically over a scan range of $3.5^{\circ}$ to $60^{\circ} 2 \theta$, scanning in steps of $2^{\circ}$ for $90 \mathrm{~s}$ per step. The samples were held between acetate foils. The theoretical patterns were generated from the crystallographic information file downloaded from the Cambridge Structural Database (CSD) - CSD reference code PYRAZB21 - using the THEO function on the Stoe WinXPOW software package. Figure 2 shows a typical example of a PXRD pattern of a phenacetin batch with the theoretical pattern overlaid, with excellent correspondence between the observed and theoretical patterns. 


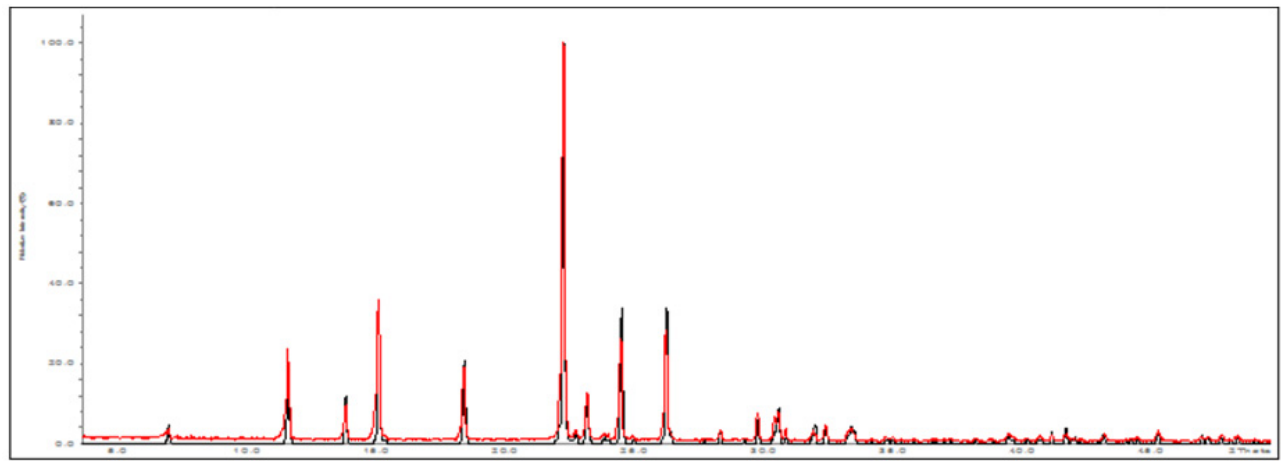

Fig. 2. Comparison of the theoretical PXRD pattern of phenacetin (black) generated from single crystal data (Hansen, 2006; CSD reference code PYRAZB21) overlaid with the experimental pattern (red) of a batch of phenacetin crystallized from ethanol.

\section{Results and discussion}

\subsection{Cooling with feedback control}

The metastable zone width (MSZW) of phenacetin from ethanol was first found by a heat / cool experiment in which the concentration was diluted after each heat /cool step until enough data points were collected to plot a solubility/super-solubility diagram. Figure 3 is the experiment progression diagram, illustrating the alternate heating and cooling cyclic programme from which the data points were collected, and Figure 4 is the solubility/supersolubility diagram of phenacetin in ethanol.

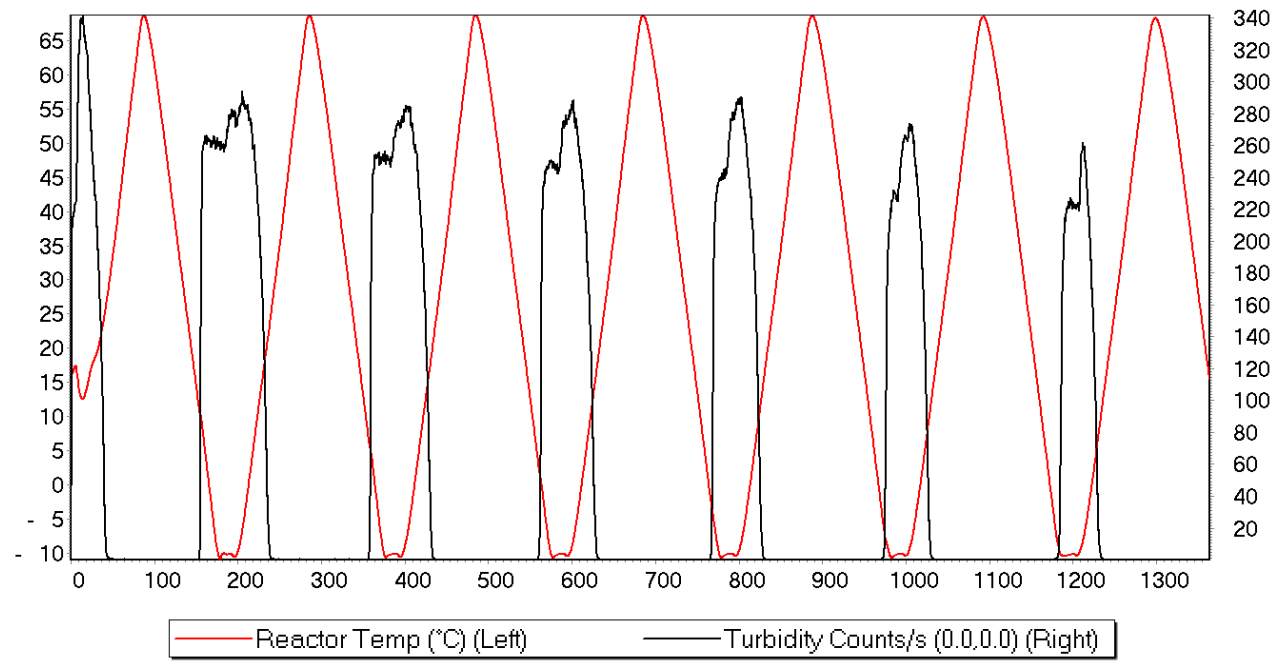

Fig. 3. The experiment progression diagram for measuring the metastable zone width (MSZW) of phenacetin in ethanol. 


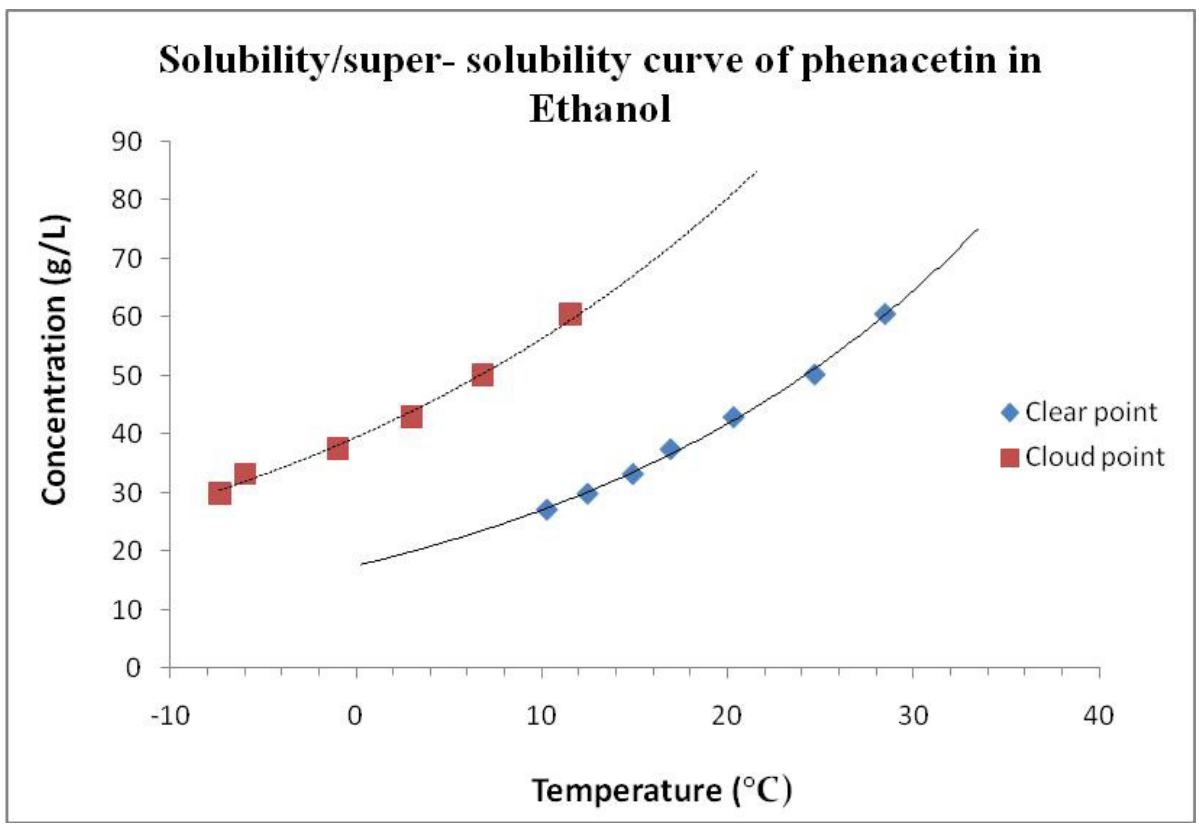

Fig. 4. The solubility/super-solubility diagram of phenacetin from ethanol.

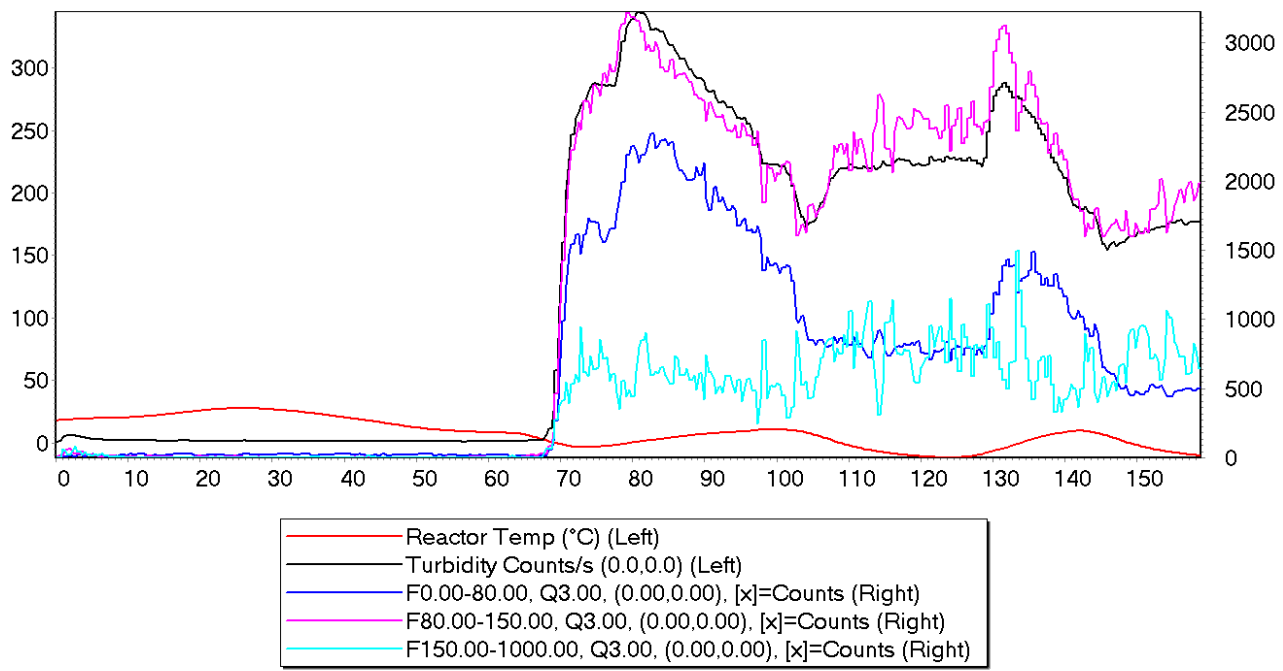

Fig. 5. The progression diagram of a heat/cool experiment of phenacetin in ethanol using turbidity counts as a termination clause $(\mathrm{F}$ is the number of particles inside the given size range - e.g., the size range $0.00-80.00$ is the content between $0-80 \mu \mathrm{m}-$ and $\mathrm{Qz}(\mathrm{x})$ is the lessthan-size for parameter $x$ and weight $z$, where $x$ is counts and $z=3$ is interpreted as the volume equivalent distribution). 
Our first experiments were undertaken using the phenacetin crystallizations in ethanol involved controlling the crystallization using turbidity counts as a termination clause for each heating and cooling step. The first experiment involved a batch without seeding. Phenacetin, at a concentration of $50 \mathrm{~g} \mathrm{~L}^{-1}$, was dissolved in ethanol, stirred at $180 \mathrm{rpm}$ and followed by a slow cool at $-0.5^{\circ} \mathrm{C} / \mathrm{min}$ until the phenacetin crystallized out of solution. The termination clause was set on the cooling step so that the cooling was terminated when the turbidity counts exceeded a set number. In this case, the programme was set to cool until the crystal population reached a turbidity of 300 counts / s. Once the turbidity counts reached the pre-set level, the experiment began to heat again until the turbidity counts dropped below another pre-set number. In this experiment, the lower limit was set at 290 counts / s, thereby terminating the step and beginning the cooling process once again. Ideally, this cyclic heating and cooling should be continued until a steady state is reached, which should result in a crystallization process where the turbidity counts are maintained at the pre-set number. Figure 5 gives this experiment's progression diagram of phenacetin in ethanol under this regime.

Using turbidity counts as the terminating clause for controlling the temperature in crystallization batches that were not seeded proved difficult. The temperature response to this terminating clause was slow, causing large fluctuations in the turbidity counts and the fine and medium total particle counts. The coarse particle counts remained relatively stable. The crystals collected at the end of the experiment were needles of varying size. PXRD analysis proved that no new forms of phenacetin were present. Since the experiment was carried out in ethanol, the crystallization of needle shaped crystals was expected. Figure 6 is a photomicrograph of the crystals collected at the end of the experiment.

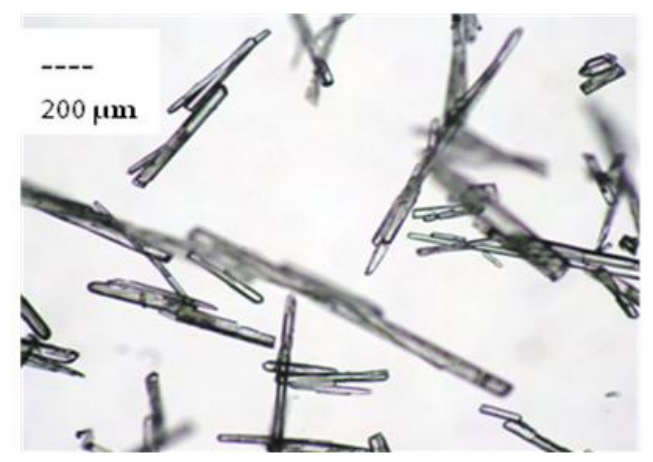

Fig. 6. Photomicrograph of crystals at the end of the experiment from a batch of a phenacetin crystallization without seeding.

It was then proposed to seed a phenacetin crystallization experiment, using the same conditions as before and with seeds of varying sizes, in order to see if better control could be achieved. It was decided to use phenacetin crystals obtained from water as the seed crystals because they typically have a plate-like habit. Any differences in habit after the crystallization of phenacetin from ethanol using these seed crystals would assist the determination of whether growth or nucleation was dominating these processes. Figure 7 is an experimental design showing the proposed heating and cooling path in the seeded experiment and Figure 8 is the experiment progression diagram of a seeded phenacetin crystallization in ethanol using turbidity counts as the termination clause. 


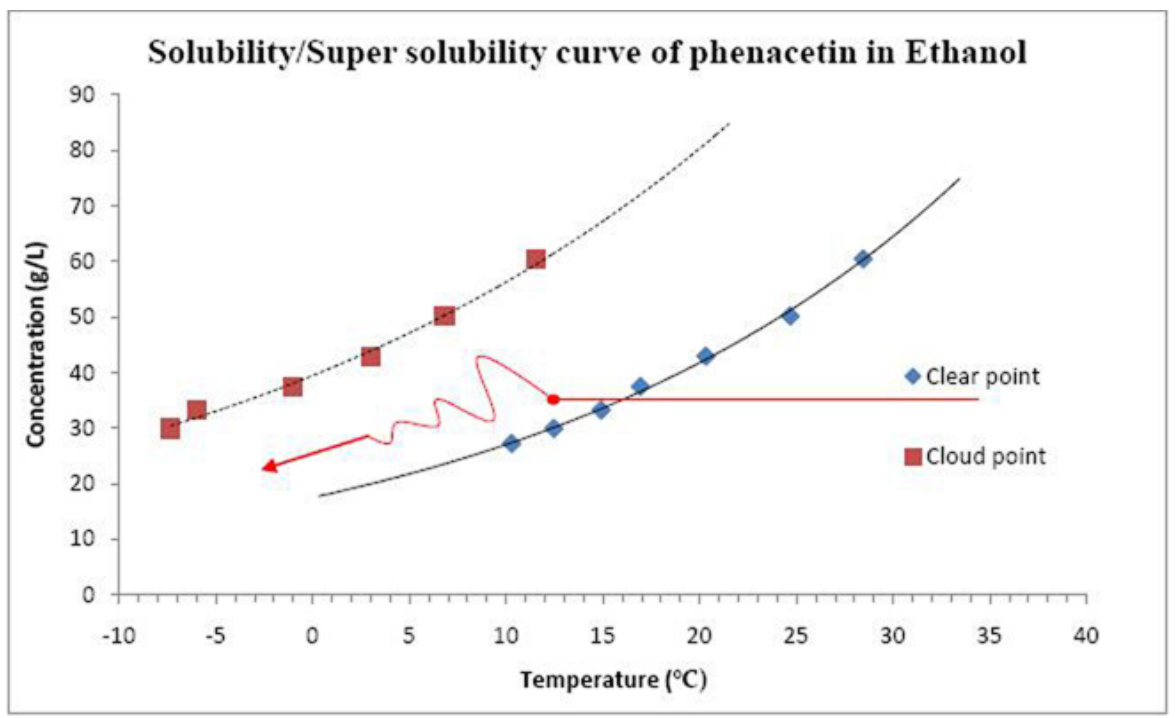

Fig. 7. Design of an experiment showing the proposed heating and cooling path using turbidity counts as the termination clause for each step (the red dot indicates the point of seeding).

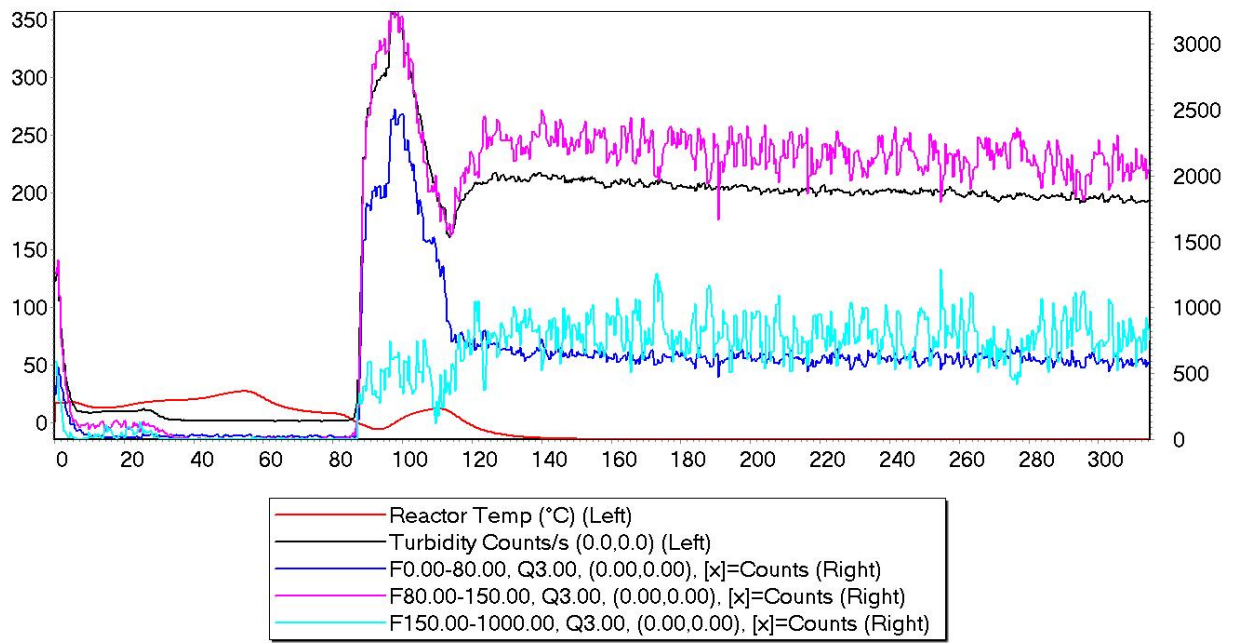

Fig. 8. The experiment progression diagram of a seeded phenacetin crystallization in ethanol using turbidity counts as a termination clause ( $\mathrm{F}$ is the number of particles inside the given size range - e.g., the size range $0.00-80.00$ is the content between $0-80 \mu \mathrm{m}$ - and $\mathrm{Qz}(\mathrm{x})$ is the less-than-size for parameter $x$ and weight $z$, where $x$ is counts and $z=3$ is interpreted as the volume equivalent distribution)

The use of turbidity counts as the terminating step to control the temperature in seeded phenacetin crystallizations resulted in a more controlled experiment. The crystallization was 
slowly cooled to within the solubility limit at a cooling rate of $-0.5^{\circ} \mathrm{C} / \mathrm{min}$. The batch was seeded with phenacetin crystals of various sizes and the crystallization was rapidly cooled at a rate of $-5^{\circ} \mathrm{C} / \mathrm{min}$. When the turbidity counts exceeded the stated level - which in this experiment was a turbidity of 200 counts / s - the process began to heat, causing the dissolution of fines and medium particles. Once the turbidity counts had reached the desired level (190 counts / s), cooling began again. The coarse and medium particles began to rise (indicating growth) while the fines remained steady. Figure 9 shows photomicrographs of the seed crystals, which are of varying size, and the end of experiment crystals, which show a more uniform crystal size and are no longer needle-like. The final crystals have a regular prismatic habit, unlike than the needles obtained from ethanol without seeding or the plates obtained from water. This reflects the operation of crystal growth from ethanol on plate-like seeds obtained from water, generating a new and more desirable prismatic habit.

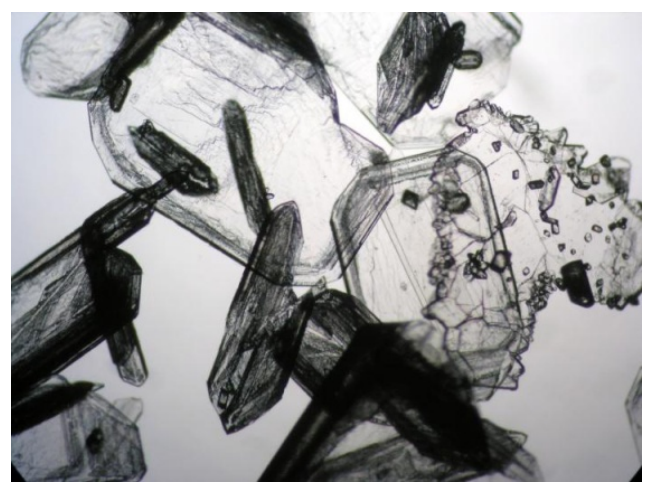

(a)

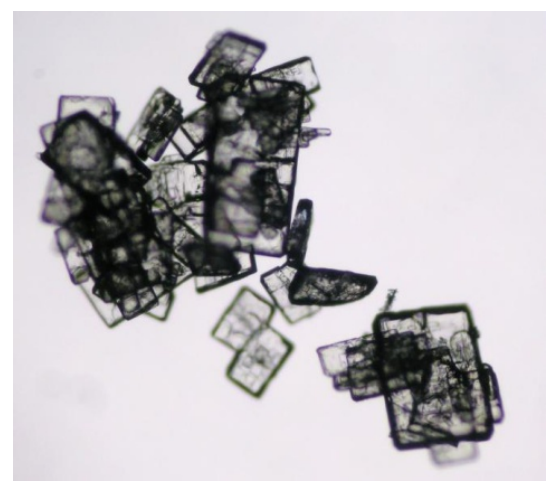

(b)

Fig. 9. Photomicrographs of (a) the seed crystals of phenacetin of varying sizes and (b) end of experiment crystals.

\subsection{Cyclic cooling}

The next series of experiments used cyclic cooling paths that had predefined temperature parameters, ensuring that a cyclic temperature profile was maintained within the MSZW limits. Rather than using the turbidity counts as a termination clause, these experiments followed plans that were programmed before the experiment began, using the data from the MSZW diagram to find the temperature limits. Figure 10 shows the design of the experiment for this crystallization.

In order to achieve the best control, it was decided to carry out a series of phenacetin crystallizations from ethanol, keeping the crystallization conditions constant throughout but varying the seed crystals. Each experiment was seeded with crystals that were sieved to give a specific size range. The three size ranges used were seed crystals under $150 \mu \mathrm{m}$, between 150-300 $\mu \mathrm{m}$ and over $500 \mu \mathrm{m}$. All of the crystallization experiments were seeded with crystals grown from water and added to an ethanol slurry using seed crystals with a weight in grams equal to $20 \%$ of the total batch concentration. Figure 11 gives the experiment progression diagram of the phenacetin crystallization from ethanol that was seeded with crystals grown from water within the size range 150-300 $\mu \mathrm{m}$. 


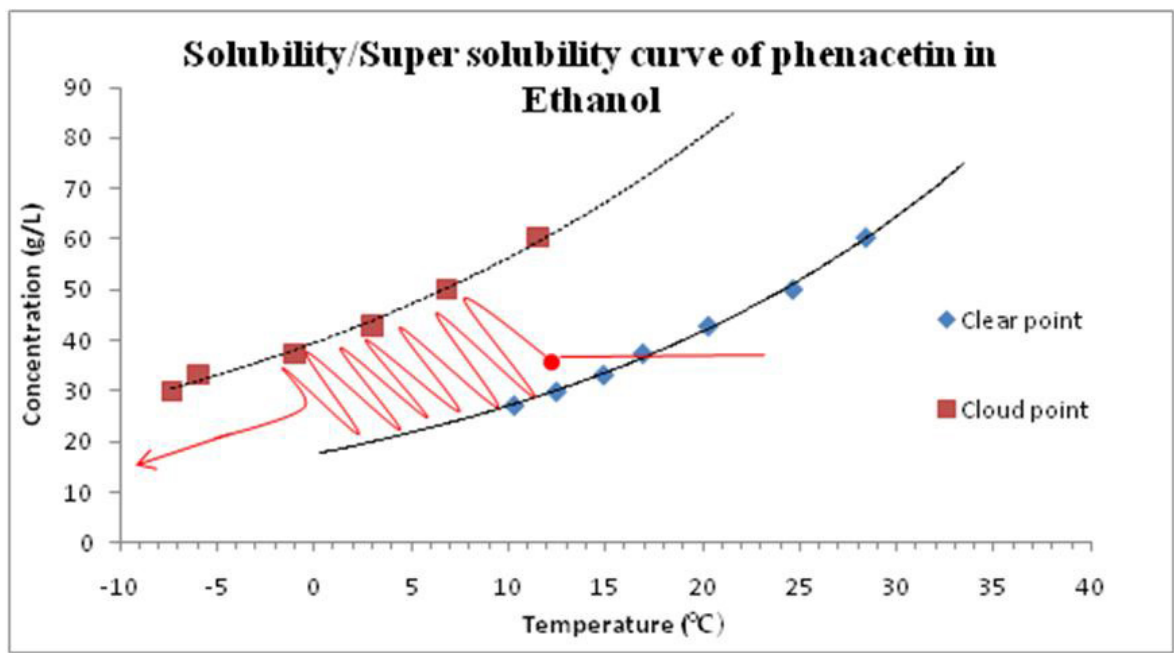

Fig. 10. An experiment design illustrating the pre-set path of the cyclic heating and cooling programme for phenacetin crystallizations in ethanol.

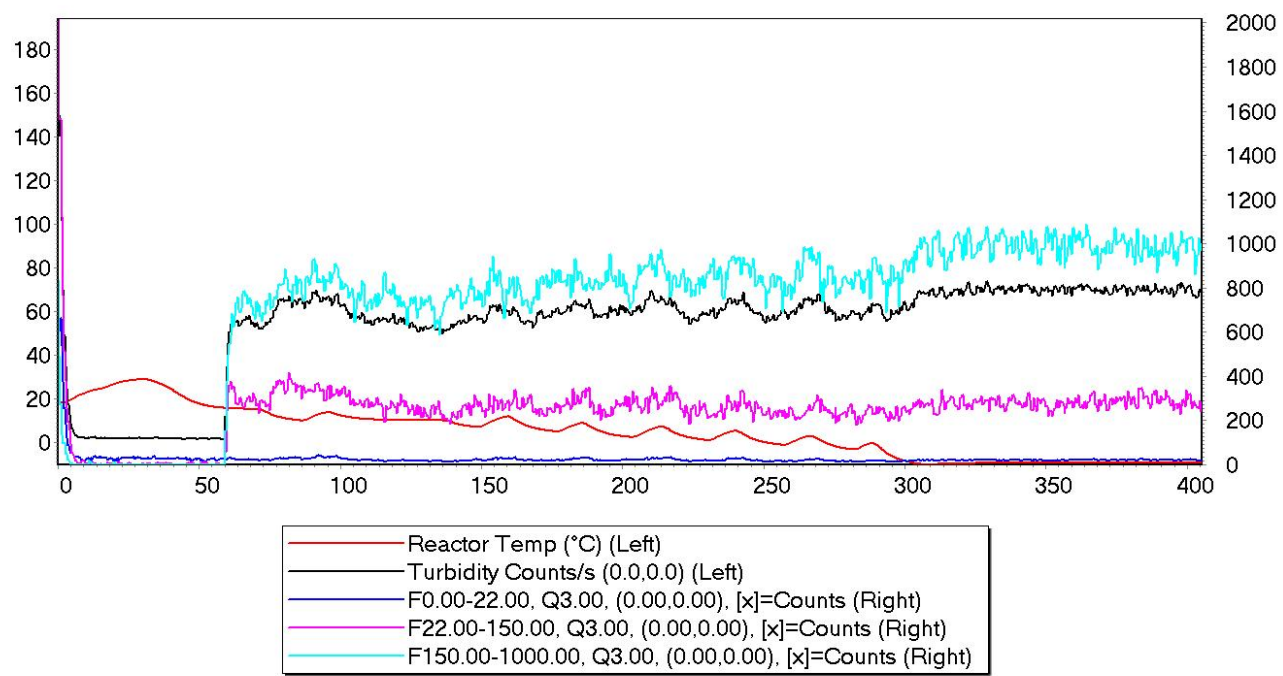

Fig. 11. Experiment progression diagram of the seeded (crystals between 150-300 $\mu \mathrm{m}$ ) phenacetin crystallization from ethanol using a pre-set cyclic plan (F is the number of particles inside the given size range - e.g., the size range $0.00-80.00$ is the content between 0 $80 \mu \mathrm{m}$ - and $\mathrm{Qz}(\mathrm{x})$ is the less-than-size for parameter $\mathrm{x}$ and weight $\mathrm{z}$, where $\mathrm{x}$ is counts and $\mathrm{z}$ $=3$ is interpreted as the volume equivalent distribution).

The experiment progression diagram shows the point of seeding as the point where there is a simultaneous increase in the turbidity counts (the black line), the medium-sized particles (the pink line indicating particles between 80-150 $\mu \mathrm{m}$ ) and the coarse particles (light blue 
line indicating particles between $150-1000 \mu \mathrm{m})$. It should be noted that at this point there is no increase in the fine particles (the dark blue line indicating particles between $0-80$ $\mu \mathrm{m})$. This is expected as the crystallization is seeded when the temperature just reaches the limit of the solubility curve, indicating a saturated solution and that spontaneous nucleation has not occurred. Therefore, the only particles in the solution are the seed crystals. Since these crystals are within the size range of $150-300 \mu \mathrm{m}$, no fine particles are present at this stage. As the solution is cooled in a cyclic heat/cool manner, any nucleation will register a rise in the counts for the fraction, indicating fine particles (the dark blue line). It can be seen from the above diagram that the dark blue line stays close to the baseline throughout the experiment, with some small intermittent increases, indicating that a small amount of nucleation occurs when the crystallization is cooling and that these fine particles are being dissolved during heating. This illustrates that this cyclic heating and cooling program is effective in controlling the nucleation process and riding the sample of fine particles, which tend to be problematic when filtering large scale crystallizations. This experiment progression diagram shows that throughout the crystallization, the mediumsized particles (the pink line) are maintained at a steady level with a slight increase in the coarse particles (the light blue) indicating growth. Figure 12 presents photomicrographs of the seed crystals grown from water and the end of experiment crystals.

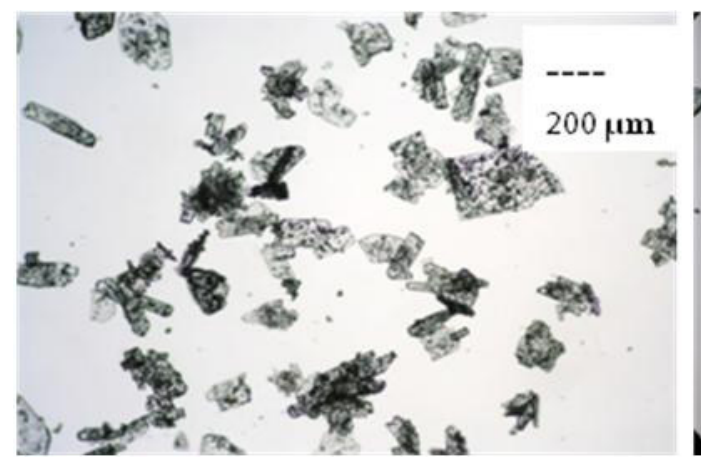

(a)

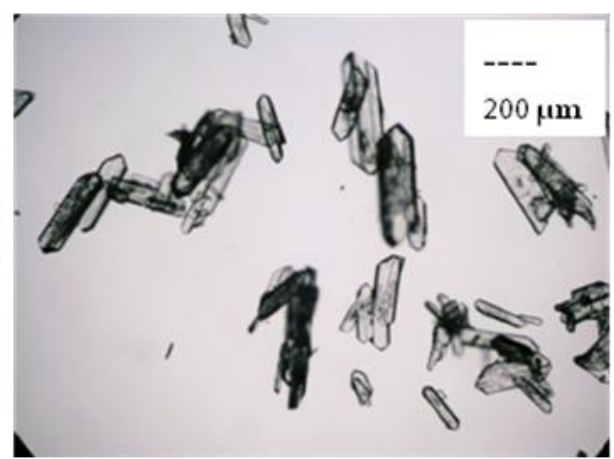

(b)

Fig. 12. Photomicrographs of (a) seed crystals between 150-300 $\mu \mathrm{m}$ and (b) end of experiment crystals.

The experiment was seeded with crystals grown from water which were plate-like in shape, and the end of experiment crystals were elongated plate-like needles. If nucleation occurs after the experiment was seeded, then these crystals will be very needle-like in shape. If the crystallization predominantly favours growth, then those seed crystals that are plate-like to begin with would have a distorted shape due to the solvent effects on the phenacetin crystals. In theory, if very fine particles precipitate during the cooling process, indicating nucleation, then these would be dissolved on the heating step. This continual heating and cooling would ensure that enough time is given to the growth of the seed crystals, while at the same time ridding the crystallization process of fine particles. The heating cycle would also ensure that the very large crystals are dissolved slightly - thereby reducing their size - the result of which would likely be a crystal batch where there is a good crystal size distribution, with the majority 
of the crystals falling under a mean crystal size. Figure 13 gives the particle size distributions of the crystals at the beginning and at the end of the experiment.

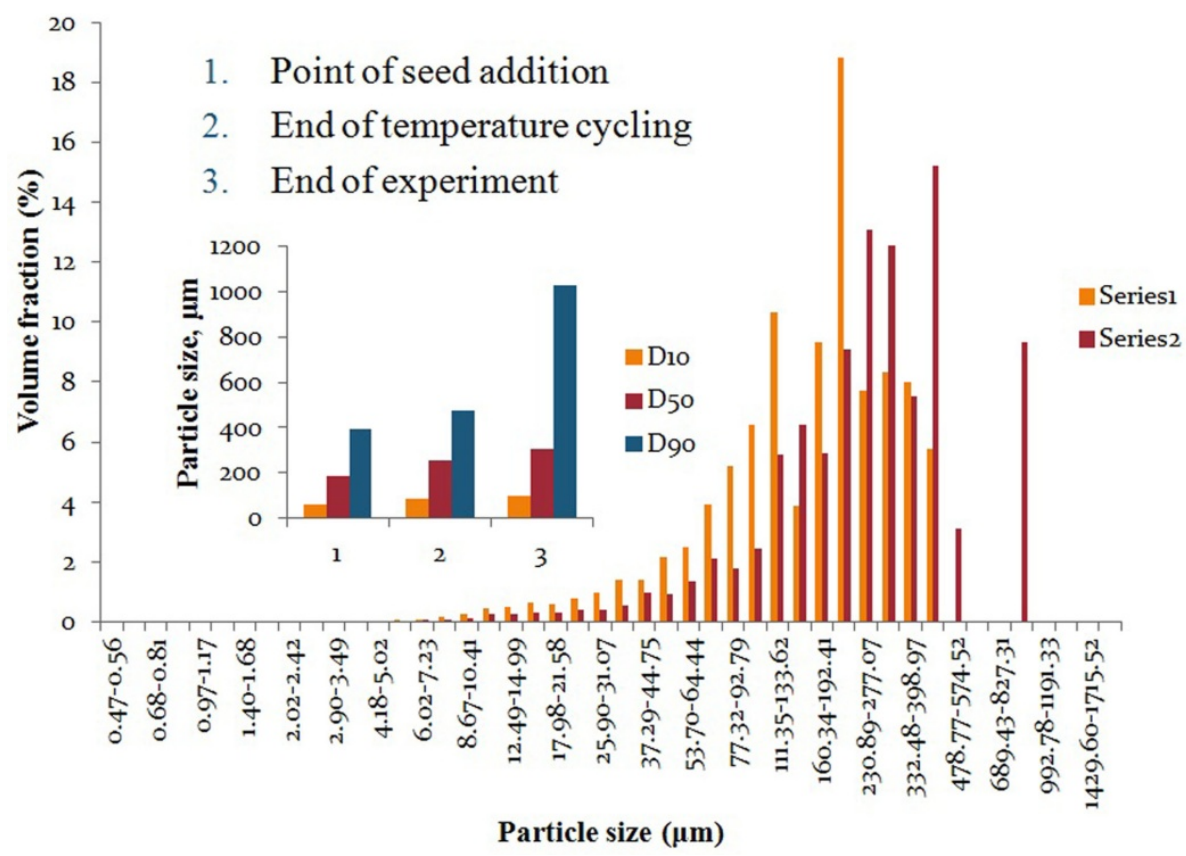

Fig. 13. Crystal size distributions of crystals shortly after the experiment is seeded (series 1) and at the end of the experiment (series 2), showing an increase in the particle size distribution and indicating growth (inset is a bar chart of the D10, D50 and D90 distributions at three points throughout the experiment).

The crystal size distributions in Figure 13 show an upward shift in the particle size from the beginning of the experiment (series 1) to the end of the experiment (series 2), which is indicative of growth. Also shown in Figure 13 are the distributions D10 (meaning the size under which $10 \%$ of the crystal population falls), D50 (the size under which $50 \%$ of the crystal population falls) and D90 (the size under which $90 \%$ of the crystal population falls). The inset of Figure 13 gives these size distributions for the crystal population at the beginning of the experiment, after the cyclic heat/cool program and at the end of the experiment. It agrees with the particle size distributions and also indicates growth. Figure 14 displays photomicrographs of a seed crystal and an end of experiment crystal.

The photographs of the crystals at the beginning and the end of the experiment show the growth of those seed crystals that began plate-like and then grew to elongated plate-like needles. The difference in size also indicates growth. When we take the seed crystal into consideration, we can see that the crystals are flat and plate-like in shape, with round edges and a rough surface. In contrast, the end of experiment crystals tend to be elongated platelike needles with a rigid, defined shape and a smooth surface. 

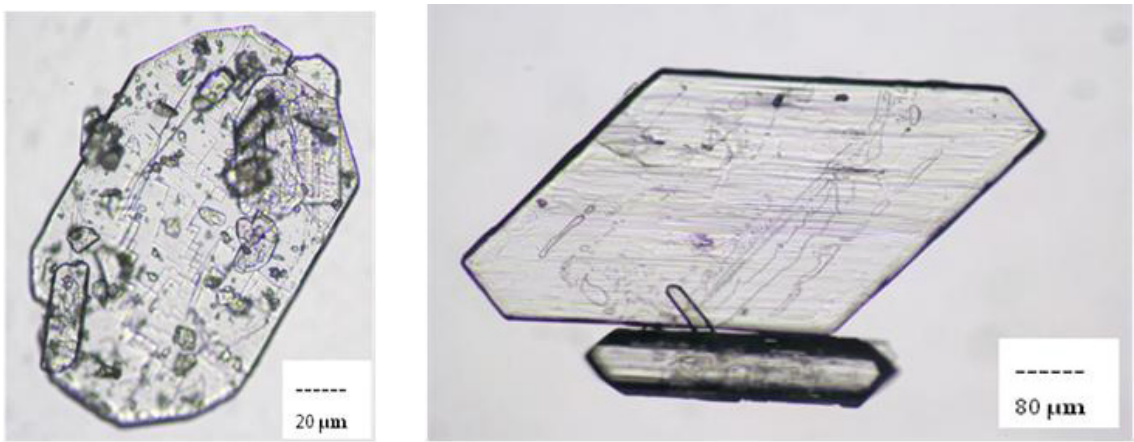

Fig. 14. Photomicrographs illustrating the difference between the seed crystals and the end of experiment crystals.

This experiment was repeated using the same experimental conditions and seeding with crystals (grown from water) under $150 \mu \mathrm{m}$. Figure 15 gives the experiment progression diagram of this crystallization. The experiment progression diagram in Figure 15 shows the point of seeding at the point where the turbidity counts (the black line) and the fine (the dark blue line), medium (the pink line) and coarse (the light blue line) particles increase simultaneously. During the cyclic heating and cooling, there is a steady decrease in the fine particle counts (the dark blue line) and a steady increase in the coarse particle counts (the light blue line), both trends indicating growth. The medium particle counts (pink line) remained steady. Figure 16 displays photomicrographs of the seed crystals under $150 \mu \mathrm{m}$ and the end of experiment crystals. Once again, the crystals grow to form plate-like needles at the end of the experiment.

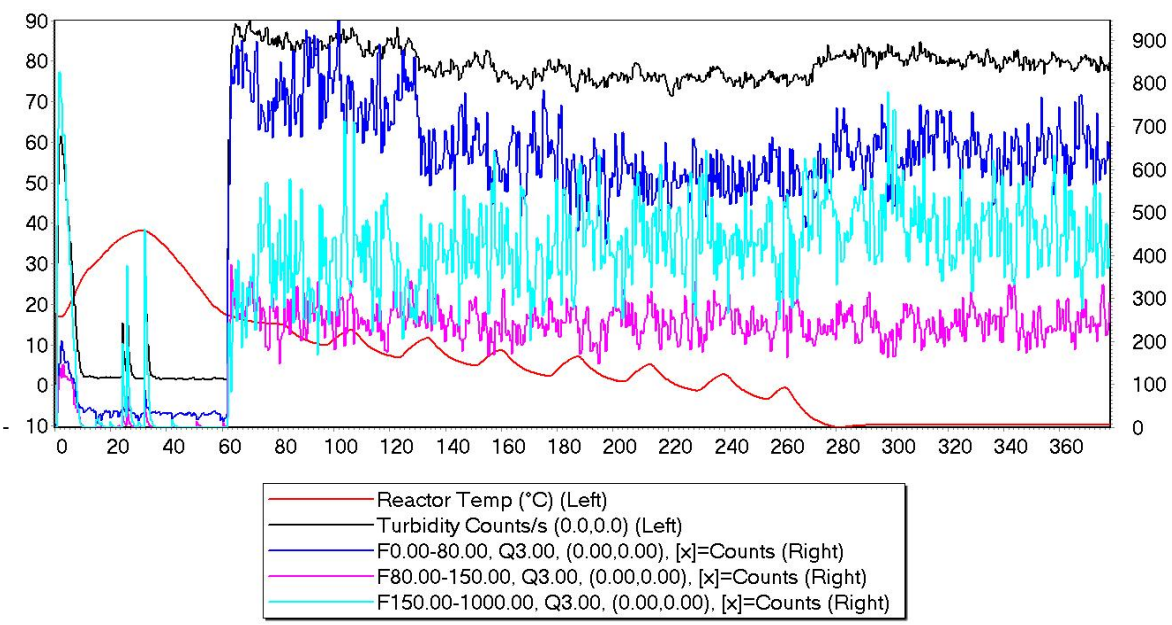

Fig. 15. The experiment progression diagram of a pre-set cyclic heating and cooling crystallization that was seeded with crystals under $150 \mu \mathrm{m}$, grown from water ( $\mathrm{F}$ is the number of particles inside the given size range - e.g., the size range $0.00-80.00$ is the content between $0-80 \mu \mathrm{m}$ - and $\mathrm{Qz}(\mathrm{x})$ is the less-than-size for parameter $\mathrm{x}$ and weight $\mathrm{z}$, where $\mathrm{x}$ is counts and $\mathrm{z}=3$ is interpreted as the volume equivalent distribution). 


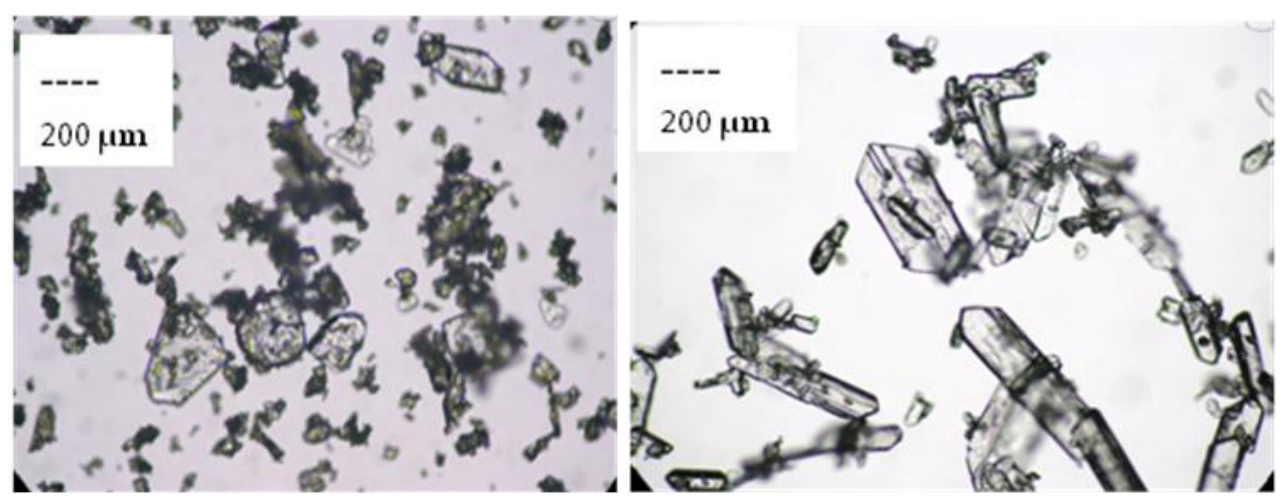

Fig. 16. Photomicrographs of (left) seed crystals grown from water under $150 \mu \mathrm{m}$ and (right) end of experiment crystals, which are plate-like needles.

Figure 17 is a bar chart of the D10, D50 and D90 particle sizes at various points in the crystallization process, which indicates the growth of the crystal. Figure 18 shows particle size distributions of the crystal population at various points in the phenacetin crystallizations seeded with crystals under $150 \mu \mathrm{m}$, with the cumulative distribution in the inset. Both of these distributions show an increase in particle size throughout the experiment, indicating growth.

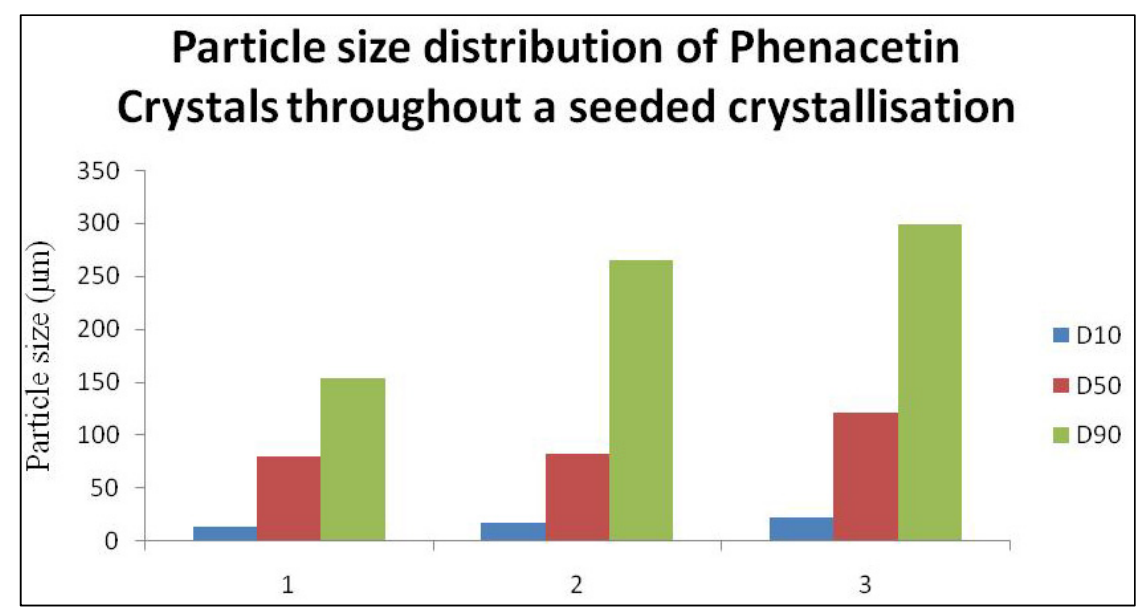

Fig. 17. A bar chart of the D10, D50 and D90 particle sizes of phenacetin crystals throughout the seeded experiments, with the seed crystals grown from water and under $150 \mu \mathrm{m}$.

The final experiment in this series used phenacetin crystallizations seeded with particles over $500 \mu \mathrm{m}$. Figure 19 is the experiment progression diagram of this crystallization. In this experiment progression diagram, the point of seeding is when the turbidity counts (the black line) and the coarse particle counts (the light blue line) increase simultaneously. Throughout the crystallization, the fine particle counts (the dark blue line) and the mediumsized particle counts (the pink line) pepper the base line, showing control over nucleation 


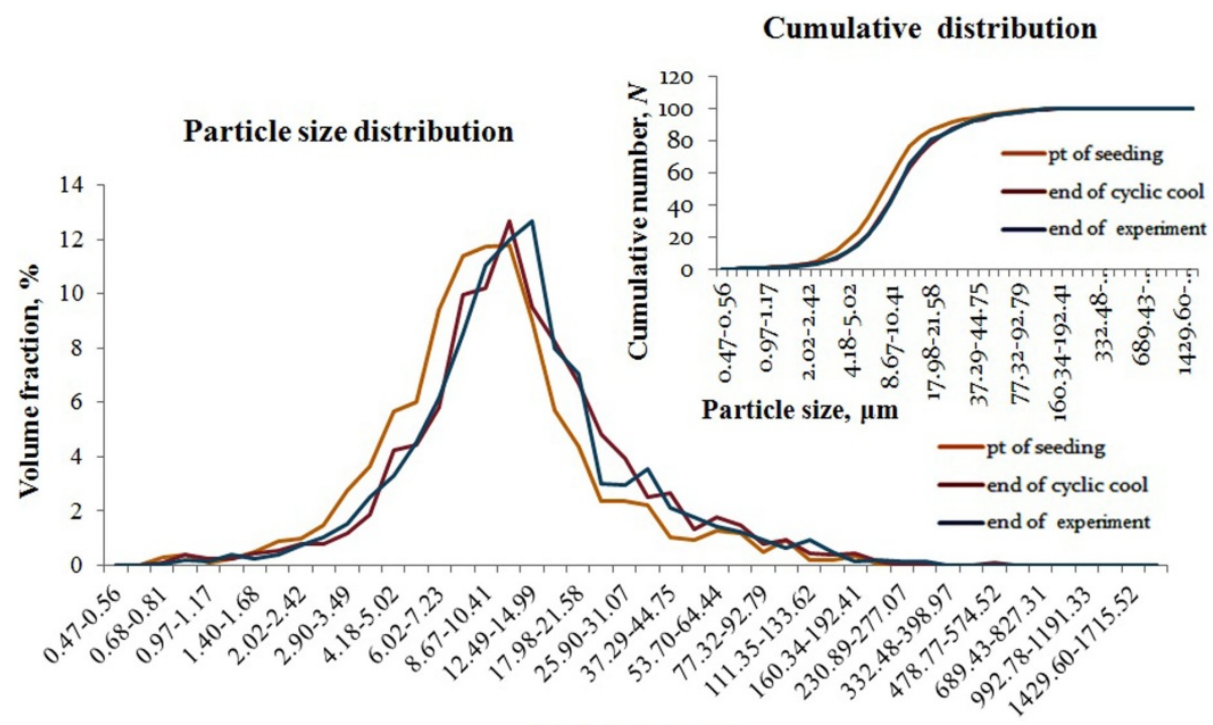

Particle size, $\mu \mathrm{m}$

Fig. 18. Particle size distributions taken throughout a seed crystallization seeded with crystals under $150 \mu \mathrm{m}$ and grown from water.

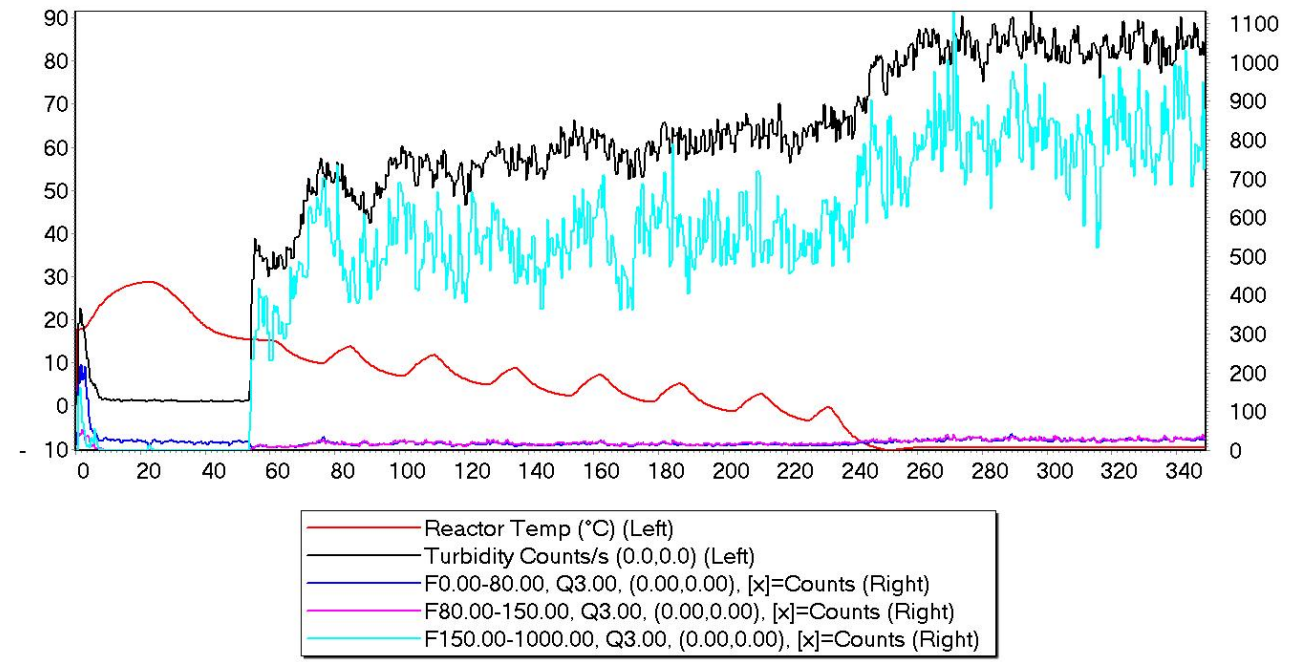

Fig. 19. Experiment progression diagram of a cyclic heating and cooling phenacetin crystallization seeded with crystals over $500 \mu \mathrm{m}$, grown from water ( $\mathrm{F}$ is the number of particles inside the given size range - e.g. the size range 0.00-80.00 is the content between 0 $80 \mu \mathrm{m}$ - and $\mathrm{Qz}(\mathrm{x})$ is the less-than-size for parameter $\mathrm{x}$ and weight $\mathrm{z}$, where $\mathrm{x}$ is counts and $\mathrm{z}$ $=3$ is interpreted as the volume equivalent distribution). 
and which is maintained at a very low level. Figure 20 shows photomicrographs of seed crystals and end of experiment crystals showing that little growth has occurred. The platelike crystals from the water become sharper around the edges, showing the change in shape from the plates to plate-like needles due to the solvent interaction.

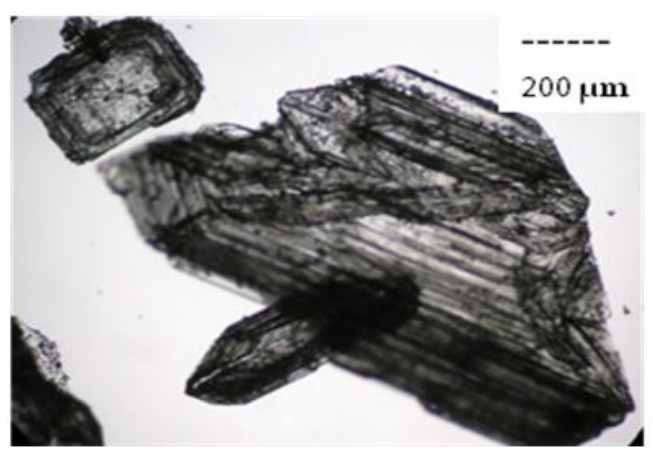

(a)

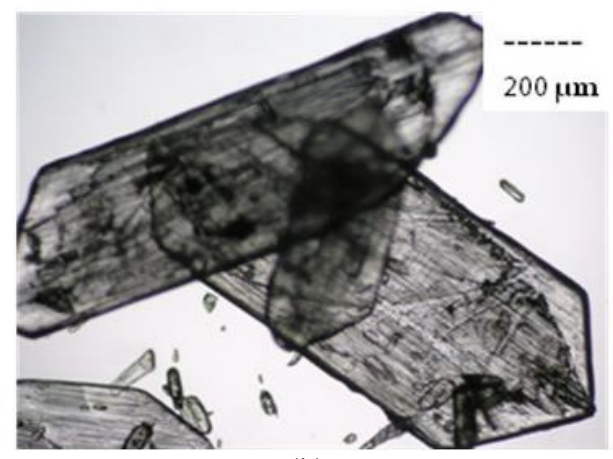

(b)

Fig. 20. Photomicrographs of (a) seed crystals over $500 \mu \mathrm{m}$, grown from water and (b) platelike needles at the end of experiment.

Figure 21 displays particle size distributions of phenacetin crystallizations seeded with crystals over $500 \mu \mathrm{m}$, indicating that little growth occurs throughout the crystallization with relatively little change in particle size distribution. The cumulative distribution in the inset also indicates this lack of growth.

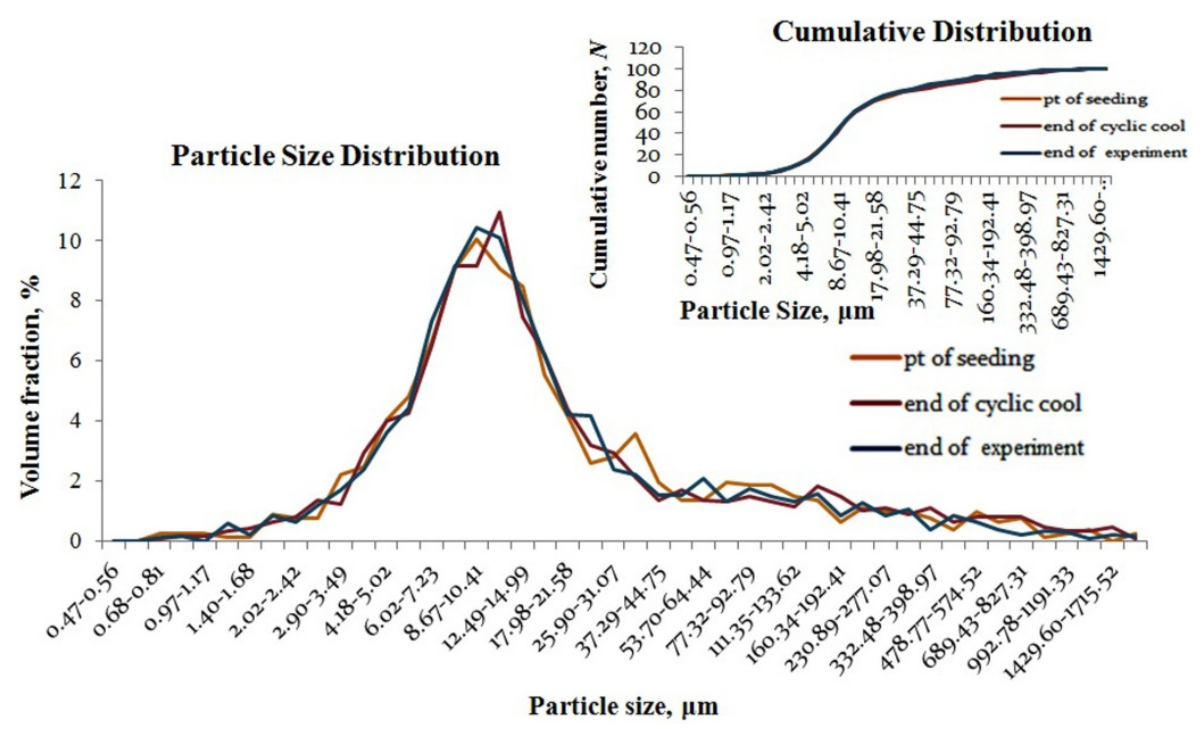

Fig. 21. Particle size distribution of the crystal population at the beginning, after cyclic heat/cool and at the end of the experiment, seeded with crystals over $500 \mu \mathrm{m}$ (inset is the cumulative distribution at the same points, indicating that little growth is occurring). 
The crystal size distributions indicate that little or no growth is taking place in this crystallization, which is not too surprising as the crystals were quite large initially and may have been at their optimum size.

The series of experiments of phenacetin crystallizations using seed crystals grown from water resulted in particle size distributions that were easy to interpret. We now wanted to see how these particle size distributions looked when they were of a crystal population which contained crystals with different dimensions, such as needles. This experiment involved phenacetin crystallizations in ethanol, using seed crystals grown from ethanol. The experiment progression diagram is given in Figure 22.

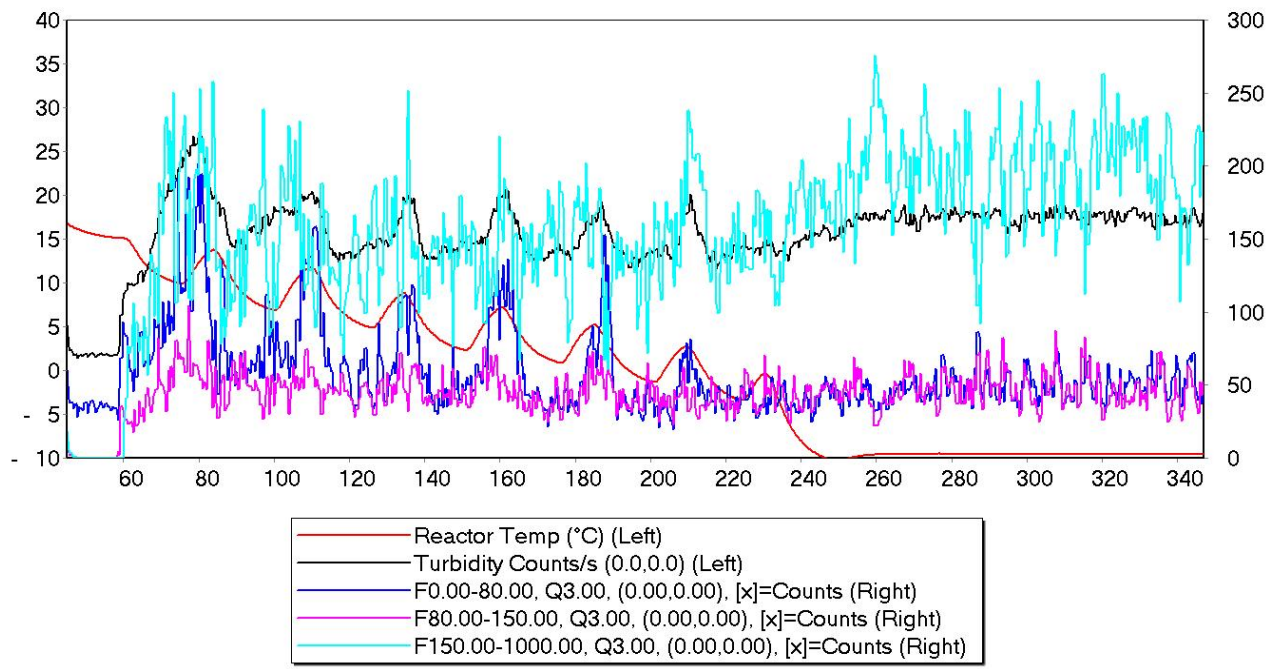

Fig. 22. Experiment progression diagram of phenacetin crystallization seeded with crystals grown from ethanol ( $\mathrm{F}$ is the number of particles inside the given size range - e.g., the size range 0.00-80.00 is the content between $0-80 \mu \mathrm{m}$ - and $\mathrm{Qz}(\mathrm{x})$ is the less-than-size for parameter $\mathrm{x}$ and weight $\mathrm{z}$, where $\mathrm{x}$ is counts and $\mathrm{z}=3$ is interpreted as the volume equivalent distribution). 
The experiment progression diagram shows a general trend where the fine particle counts (the dark blue line) and medium-sized particle counts (the pink line) maintain a low level and where there is a rise in the coarse particle counts (the light blue line). In Figure 22, the reason for the significant noise in the measurement is likely to be due to the shape of the needles and how they are interpreted as they pass the window of the laser probe. The longest face along the length of a needle may be interpreted as a large particle, while the narrow face at either end of the needle may be interpreted as a fine particle. Thus, every time this needle moves past the window of the laser probe, depending on its orientation, it may be assigned a different particle size leading to significant noise in the measurement. Figure 23 displays photomicrographs of the seed crystals and the end of experiment crystals.

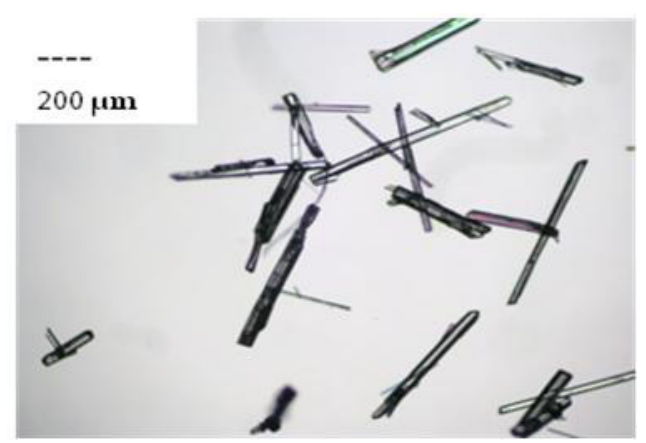

(a)

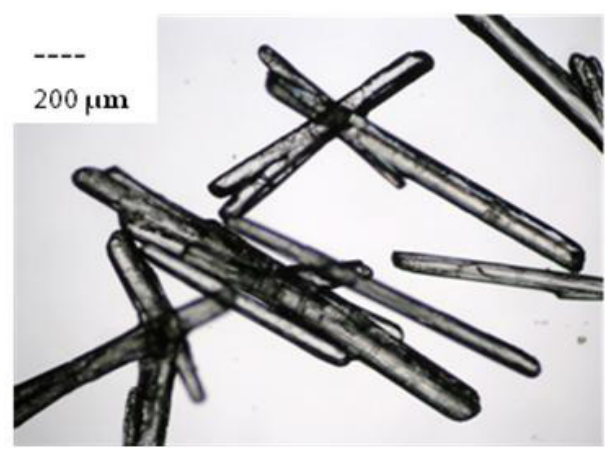

(b)

Fig. 23. Photomicrographs of (a) seed crystals grown from ethanol and (b) end of experiment crystals.

These photomicrographs indicate growth with the crystals maintaining their needle-like shape, as would be expected. Figure 24 shows particle size distributions of the crystal population at various points throughout the crystallization. 


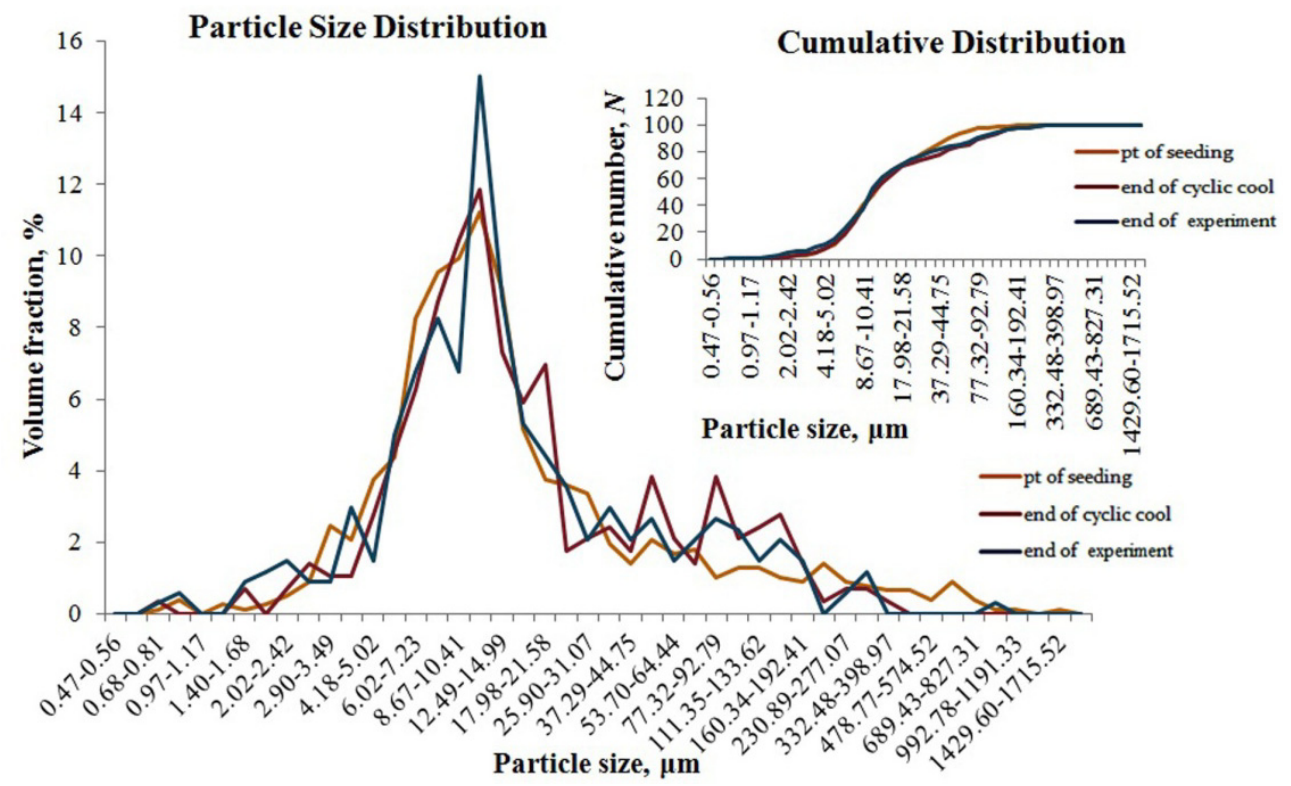

Fig. 24. Particle size distributions of phenacetin crystal populations at various points in a crystallization seeded with crystals grown from ethanol (inset is the cumulative crystallization at the same points).

The particle size distributions indicate growth, as there is an upward shift in particle size throughout the crystallization. These distributions also indicate an environment where there are many differing crystals sizes, as can be seen from the multimodal nature of the trend lines. This is most likely explained by differing orientations of the needle-like crystals as they pass the window of the laser probe. The laser sees the crystal as a two dimensional shape and measures the longest chord length. When the crystal shape is needle-like, the population of crystals passing the sensor can appear as one of the crystals with many varying sizes, depending upon the orientation of the crystals (Barthe, 2008). This is a negative aspect of in situ laser probe technology and makes the particle size distributions more difficult to interpret.

The use of seeded batch cooling crystallizations with temperature cycling may be preferable for the control of nucleation and growth, enhancing the size uniformity of the crystals. Cyclic temperature cooling programmes may be preferable to linear temperature cooling programs for the size uniformity of crystals (Lindenberg et al., 2009). Single crystal analysis and microscopy can be used to follow the direction of the growth of seed crystals during a crystallization process.

\section{Conclusions}

Crystallizations of phenacetin from ethanol were carried out in a 1 L HEL Autolab vessel fitted with a HEL Lasertrack in situ particle sizing laser probe. Two forms of modified cooling regimes were examined, each of which was intended to provide a narrower range of 
crystal sizes and shapes rather than a straightforward linear cool. The first cooling regime involved using turbidity counts as a termination clause for each heating and cooling step. The termination clause was set on the cooling step, so that the cooling was terminated when the turbidity counts exceeded a set number. Once the turbidity counts reached the pre-set level, the experiment began to heat again until the turbidity counts dropped below another pre-set number. Using this approach for crystallization batches that were not seeded proved difficult. The temperature response to this terminating clause was slow, causing large fluctuations in the turbidity counts and the fine and medium total particle counts. The use of turbidity counts as the terminating step for controlling temperature in seeded phenacetin crystallizations resulted in a more controlled experiment, giving more uniform crystals. It was also possible to generate much more prismatic crystals in this way, in contrast to the highly needle-like crystals obtained without seeding.

The second approach used cyclic cooling paths that had predefined temperature parameters, ensuring that a cyclic temperature profile was maintained within the MSZW limits. Each experiment was seeded with crystals that were sieved to give specific size ranges of less than $150 \mu \mathrm{m}$, between 150-300 $\mu \mathrm{m}$ and over $500 \mu \mathrm{m}$. The use of the seeds in the 150-300 $\mu \mathrm{m}$ range gave relatively uniform elongated plates of good quality. The use of seeds under 150 $\mu \mathrm{m}$ gave rise to elongated plates of reasonable uniformity. Those seeds over $500 \mu \mathrm{m}$ grew the least during the cooling process and were the least effective in generating uniformity of size and shape, possibly due to these seed crystals' being so large that they had potentially already reached their optimum size. Overall, the pre-programmed cyclic cooling regime was found to be easier to operate and provided good quality crystal batches when used in conjunction with sieved seeds.

\section{Acknowledgements}

The authors are grateful for financial support from the Solid State Pharmaceutical Cluster (Science Foundation Ireland grant 07/SRC/B1158).

\section{References}

Abu Baker, M. R.; Nagy, Z. K.; Tan, R. B. H.; Saleemi, A. N. \& Rielly, C. D. (2009) The Impact of Direct Nucleation Control on Crystal Size Distribution in Pharmaceutical Crystallization Processes. Crystal Growth and Design, 9, 1378-1384

Abu Bakar, M. R.; Nagy, Z. K. \& Rielly, C. D. (2009) Seeded Batch Cooling Crystallization with Temperature Cycling for the Control of Size Uniformity and Polymorphic Purity of Sulfathiazole Crystals. Organic Process Research and Development, 13, 1343-1356

Barthe, S. C. (2008) Investigation and modeling of the mechanisms involved in batch cooling crystallization and polymorphism through efficient use of the FBRM. PhD Thesis, Georgia Institute of Technology, 119-138

Garside, J.; Gaska, C. \& Mullin, J. W. (1972) Crystal Growth Rate Studies with Potassium Sulphate in a Fluidized Bed Crystallizer. Journal of Crystal Growth, 13/14, 510-516

Griffiths, H. (1925) Mechanical Crystallization. J. Soc. Chem. Ind. Trans., 44, 7T-18T

Hansen, L. K.; Perlovich, G. L. \& Bauer-Brandl, A. (2006) Redetermination of pethoxyacetanilide (phenacetin). Acta Crystallographica, E62, o2712-o2713 
Lindenberg, C.; Krattli, M.; Cornel, J.; Mazzotti, M. \& Brozio, J. (2009) Design and Optimization of a Combined Cooling/Antisolvent Crystallization Process. Crystal Growth and Design, 9, 1124-1136

Prescott, L. F. (1980) Kinetics and metabolism of paracetamol and phenacetin. British Journal of Clinical Pharmacology, 10, 291-298

Woo, Xing Yi; Nagy, Z. K.; Tan, R. B. H. \& Braatz, R. D. (2009) Adaptive Concentration Control of Cooling and Antisolvent Crystallization with Laser Backscattering Measurement. Crystal Growth and Design, 9, 182-191 
(C) 2012 The Author(s). Licensee IntechOpen. This is an open access article distributed under the terms of the Creative Commons Attribution 3.0 License, which permits unrestricted use, distribution, and reproduction in any medium, provided the original work is properly cited. 